\title{
Methanol Poisoning Leading to Brain Death: A Case Report
}

\author{
Jakub Glowala1* , Jeremy B. Richards² \\ ${ }^{1}$ McGaw Medical Center of Northwestern University, Chicago, IL, USA \\ 2 Beth Israel Deaconess Medical Center, Boston, MA, USA
}

\begin{abstract}
Introduction: The COVID-19 pandemic has put increased stress on medical systems, infrastructure, and the public in expected and unexpected ways. This case report summarises an unexpected case of methanol poisoning from hand sanitiser ingestion due to changes in industry regulations, increased demand for cleaning products and severe psychosocial stressors brought on by the pandemic. Severe methanol toxicity results in profound metabolic disturbances, damage to the retina and optic nerves, and potentially death. Case Presentation: The patient was a 26-year-old male with alcohol use disorder who presented with one day of nausea, vomiting, and abdominal pain after consuming hand sanitiser. Within a few hours, the patient had suffered multiple seizures, cardiac arrests and required admission to the ICU for emergent management of methanol poisoning. EEG and brain perfusion imaging were performed to confirm brain death, given concerns about the cranial nerve exam after methanol poisoning. Conclusions: While rare, methanol toxicity remains a potentially fatal poisoning in the United States and worldwide. When healthcare and public resources are strained, healthcare professionals must consider particularly abnormal presentations. In patients suspected of brain death from methanol toxicity, cranial nerve examination may be unreliable. Therefore, additional testing is necessary to confirm brain death.
\end{abstract}

Keywords: methanol toxicity, brain death

Received: 6 March 2021 / Accepted: 10 October 2021

\section{INTRODUCTION}

The COVID-19 pandemic has transformed the modern world, and many of the indirect harms to patients will only become evident in retrospect. For patients with alcohol use disorder, the pandemic has increased alcohol-seeking behaviours and alcohol consumption and increased withdrawal rates due to decreased access to ethanol [1]. In addition, with the increased use of harsh cleaning agents, strained production lines potentially vulnerable to contamination, and messaging advocating consumption of potentially lethal substances, patients may be particularly susceptible to poisoning [2].

This case describes an atypical source of methanol toxicity in a young male with alcohol use disorder. It highlights the need for a pathophysiologic approach to clinical medicine during times of uncertainty. It also emphasises the need to avoid anchoring even if there is a known source of poisoning and highlights the difficulty of interpreting neurologic examinations in the setting of methanol toxicity.

\section{CASE DESCRIPTION}

A 26-year-old male with alcohol use disorder was brought to Emerson Hospital, Concord, MA, USA, in the early morning for evaluation. The patient suffered from nausea, vomiting, and abdominal pain after drinking multiple bottles of hand sanitiser on the previous day. On arrival, he was confused and appeared unwell but was able to confirm he had ingested hand sanitiser and no other substances.

In the Emergency Department, he rapidly worsened, becoming unresponsive and suffered multiple seizures. He then suffered five brief cardiac arrests, with the return of spontaneous circulation following administration of epinephrine and bicarbonate, all within the first few hours of presentation.

He was intubated after this final cardiac arrest, approximately 30 minutes after becoming unresponsive; he had a Glasgow Coma Scale (GCS) of 3, accompanied by seizures and several cardiac arrests. A head and abdominal computerised tomography scan were 
undertaken immediately but showed no particular abnormalities.

Laboratory studies (Table 1) demonstrated a severe anion gap metabolic acidosis and hyperosmolality. $\mathrm{He}$ was initiated on norepinephrine $(0.5 \mathrm{mg} / \mathrm{mL}$ administered at $0.5 \mathrm{mcg} / \mathrm{kg} / \mathrm{min}$ IV [Hospira, Inc., Lake Forest, IL, USA]), phenylephrine $(20 \mathrm{mcg} / \mathrm{mL}$ administered at $3 \mathrm{mcg} / \mathrm{kg} / \mathrm{min}$ IV [Eton Pharmaceuticals, Deer Park, IL, USA]), vasopressin (20 units/mL administered at 2.4 units/hr IV [Par Pharmaceutical, Chestnut Ridge, NY, USA]), and epinephrine $(4 \mathrm{mcg} / \mathrm{L}$ administered at $0.1 \mathrm{mcg} / \mathrm{kg} / \mathrm{min}$ IV [Par Pharmaceutical, Chestnut Ridge, NY, USA]).

Over the following few hours, five litres of intravenous fluids were administered for persistently low blood pressures and mean arterial pressures, monitored with an arterial catheter placed at the time of intubation.

He was initiated on fomepizole $(1 \mathrm{gm} / \mathrm{mL}$ administered at $15 \mathrm{mg} / \mathrm{kg}$, followed by $10 \mathrm{mg} / \mathrm{kg}$ every 12 hours

Table 1: Summary of patient's lab values on arrival to the ED and upon completion of dialysis.

\begin{tabular}{lcc} 
& On Arrival & Post-Dialysis \\
WBC & 17.5 & 12.3 \\
Hgb & 12.8 & 10.0 \\
Hct & 42.6 & 31.6 \\
Plt Count & 248 & 82 \\
Glucose & 83 & 144 \\
Urea & 13 & 2 \\
Creatinine & 1.9 & 0.7 \\
Na & 150 & 150 \\
K & 6.4 & 4.2 \\
Cl & 114 & 116 \\
HCO3 & $<2$ & 25 \\
Anion Gap & Unmeasurable & 9 \\
Calcium & 7.5 & 7.8 \\
Phos & 8.8 & 4.1 \\
Mg & 3.7 & 1.9 \\
Osmolality & 427 & 299 \\
Ven pO2 & 182 & 129 \\
Ven pCO2 & 44 & 36 \\
Ven pH & 6.53 & 7.49 \\
Lactate & 19.0 & 1.5 \\
ALT & 85 & 245 \\
AST & 99 & 1192 \\
Creatine Kinase & & 3140 \\
Lipase & 75 & \\
cTroponinT & & 0.01 \\
\hline
\end{tabular}

for 4 doses, then $15 \mathrm{mg} / \mathrm{kg}$ every 12 hours IV (Jazz Pharmaceuticals, Inc. Palo Alto, CA, USA).

Due to the concern about the possibility of non-ethanol alcohol toxicity, despite the patient's lack of access to any other poisons at home and denial of any other ingestions, the night of his presentation, he was transferred to an academic hospital, Beth Israel Deaconess Medical Center, Boston, USA.

On arrival, the patient's GCS was 3. He had a heart rate of $80 \mathrm{bpm}$, blood pressure $94 / 52 \mathrm{mmHg}$ on the four vasopressors described above; an oxygen saturation of $97 \%$ on volume assist-control ventilation with a tidal volume of $440 \mathrm{cc}$, respiratory rate of 34 breaths per minute, positive end-expiratory pressure of $8 \mathrm{~cm} \mathrm{H}_{2} \mathrm{O}$, and a fraction of inspired oxygen of $50 \%$. His pupils were $3 \mathrm{~mm}$ in diameter with no response to light. $\mathrm{He}$ was comatose, with no response to noxious stimuli. On the recommendation of the nephrology service, given the presence of severe acidosis, a bicarbonate infusion was started.

Fomepizole (Jazz Pharmaceuticals, Inc. Palo Alto, CA, USA) was continued, and thiamine $(100 \mathrm{mg} / \mathrm{mL}$, 500mg IV [FFF Enterprises, Temecula, CA, USA], folate (1mg tablet, 1mg PO [LGM Pharma, Boca Raton, FL, USA]), and pyridoxine $(100 \mathrm{mg} / \mathrm{mL}, 100 \mathrm{mg}$, IV [Biocare SD, Tempe, AZ, USA]) were provided, given concern for toxic alcohol ingestion.

The nephrology service was consulted early in the morning of Day 1 post-admission and he was admitted to the medical intensive care unit; emergency dialysis was commenced.

Targeted temperature management (TTM) was initially deferred given the severity of his condition.

His alcohol panel was positive for methanol but otherwise negative. In addition, his osmolar gap was serially monitored, given the relatively long processing time of methanol testing.

The morning after admission, his $\mathrm{pH}$ was 7.3 with continuous renal replacement therapy (CRRT) and a continuous bicarbonate infusion. Targeted temperature management was initiated at that time and maintained for 24 hours. Continuous renal replacement therapy was discontinued once his osmolar gap closed from an initial $118.0 \mathrm{mOsm} / \mathrm{kg}$ to $-10 \mathrm{mOsm} / \mathrm{kg}$ (normal range -14.0 to 10.0$)$.

Moreover, his methanol levels were undetectable. His metabolic derangements resolved after 24 hours 
on dialysis, he no longer required vasopressors, and his temperature was recorded at $37^{\circ}$ Celsius.

On Day 3, post-admission, a neurologic exam was performed. Brain stem reflexes were absent; repeat head CT demonstrated diffuse oedema consistent with global hypoxia and ischemia. An electroencephalogram did not demonstrate cerebral activity, and apnea testing was consistent with brain death. Despite all examination manoeuvres indicating brain death, we could not rule out that optic nerve damage from methanol toxicity was potentially obfuscating the cranial nerve exam, so a brain perfusion scan (Figure 1) was performed. This confirmed brain death.

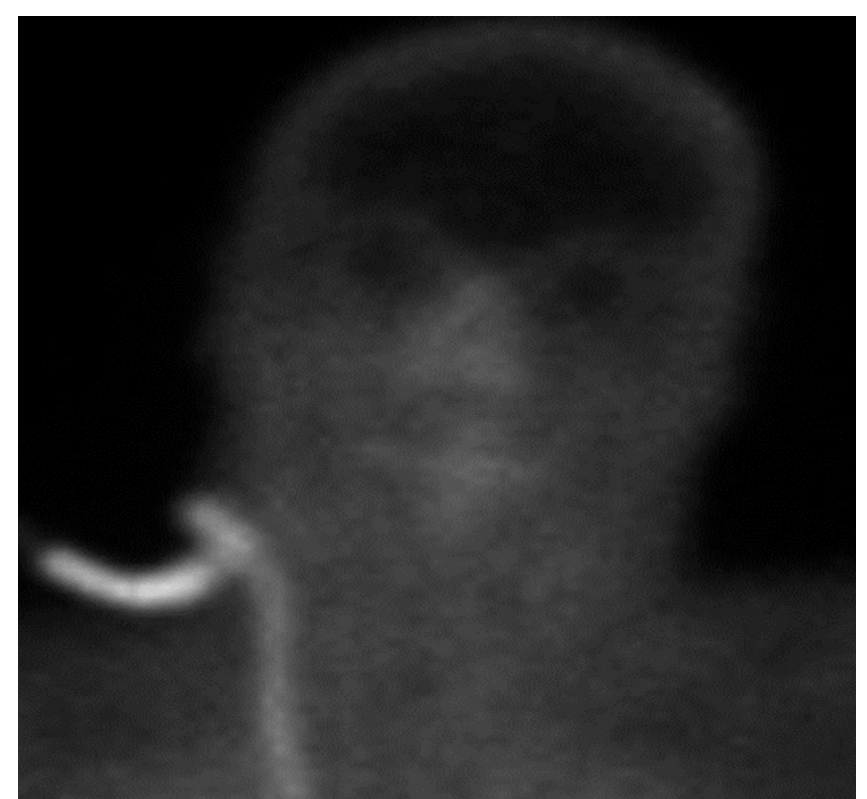

Fig. 1. Brain perfusion scan demonstrating no discernible uptake by the brain.

\section{DIscussion}

Methanol poisoning is relatively rare and potentially fatal toxicity in the United States and throughout the world. Roughly $90 \%$ of cases are unintentional ingestions, and the most common sources $(84.5 \%)$ are automotive products $[3,4]$. Though most exposures are ingestions, methanol can be toxic through oral, pulmonary, or skin contact [5]. Hand sanitiser, though not meant to be ingested, may be used by patients as a means of intoxication when ethanol is unavailable. Given the health risks associated with methanol, hand sanitisers are prohibited explicitly from containing this compound. However, given the COVID pandemic, there has been both a massive influx of new hand sanitiser manufacturers as well as the loosening of Federal Drug Administration (FDA) manufacturing oversight [6].

Given the rarity of severe presentations and the unexpected sources of exposure, this case describes key features of methanol toxicity. Methanol is a toxic alcohol that is an unmeasured osmol, elevating the osmolar gap [7]. It is metabolised to formaldehyde and then, via aldehyde dehydrogenase, to formic acid. The latter is a toxic metabolite and causes an anion-gap metabolic acidosis [7]. As a potent alcohol dehydrogenase inhibitor, fomepizole inhibits the formation of formic acid, and dialysis is used to remove methanol $[8,9]$. In addition, despite no randomised controlled trials supporting its use, folate is often provided to augment formic acid elimination, given its role as a cofactor in the formic acid oxidation pathway (Figure 2) [10].

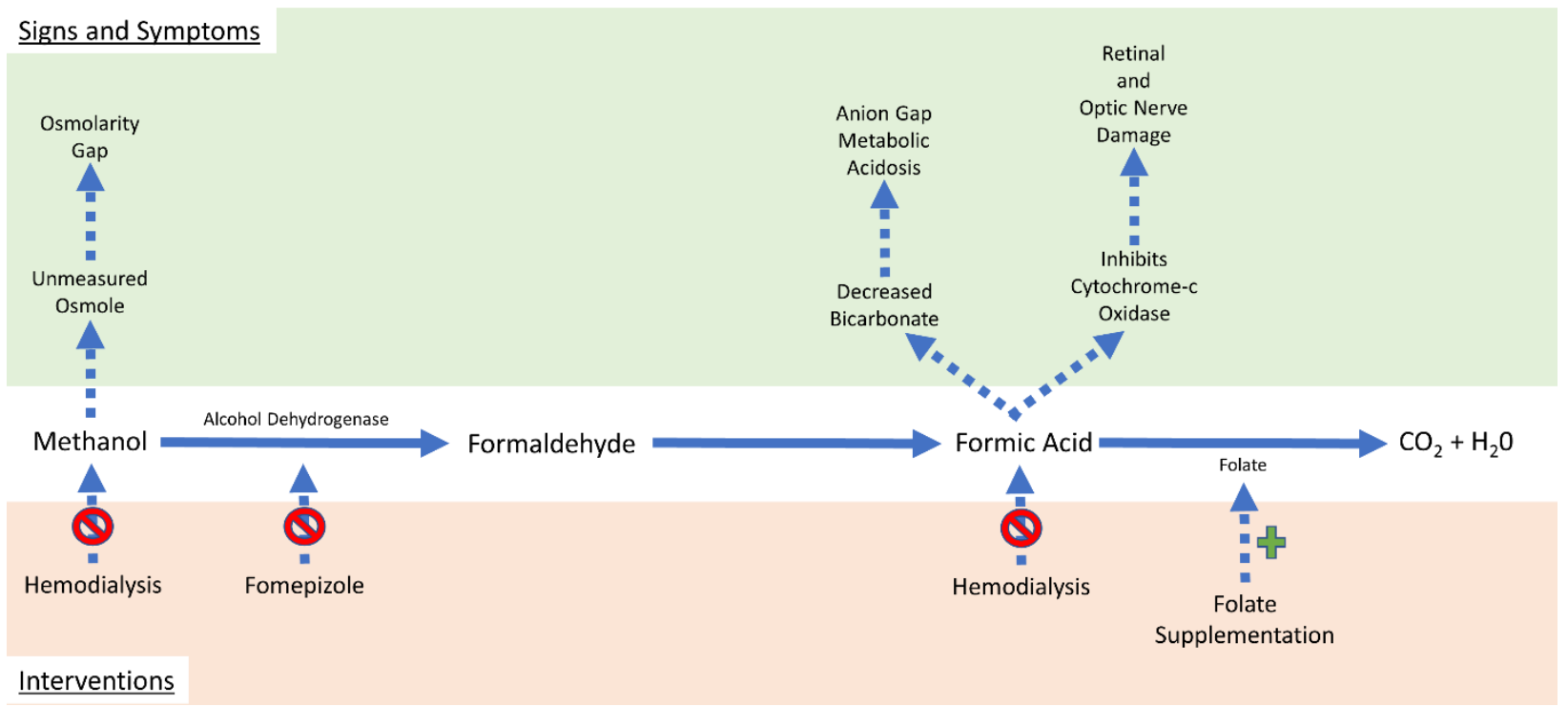

Fig. 2. Graphical summary of the methanol pathway with the resulting signs and interventions. 
Upon resolution of acute intoxication and confirmation of clearance of serum methanol, patients should be evaluated for neurologic damage, particularly blindness due to optic nerve atrophy and retinal damage [11]. In this case, the patient's severe acidosis and multiple cardiac arrests led to anoxic brain injury. Given potential retinal and optic nerve damage, it was unclear if the lack of corneal reflex was a feature of brain death or optic nerve death from methanol toxicity. Given the unreliable neurologic exam, brain perfusion imaging was used to confirm brain death.

After his death, the Federal Drug Administration issued a press release about methanol-contaminated hand sanitisers, including confirmation that the product consumed by the patient (Figure 3) contained methanol. This brand of hand sanitiser was subsequently removed from the market [12]. This highlights a key clinical principle as the significant number of contaminated hand sanitisers was unknown at the time of the patient's presentation. Furthermore, the patient had clearly ingested hand sanitiser without other possible sources of ingestion. A diagnosis could have been delayed without understanding the first principles and careful analysis of the patient's metabolic derangements. It is crucial to maintain a broad differential as toxic exposures or ingestions can come from unexpected sources. In addition, capacity strain in the setting of a global viral pandemic increases the risk of health

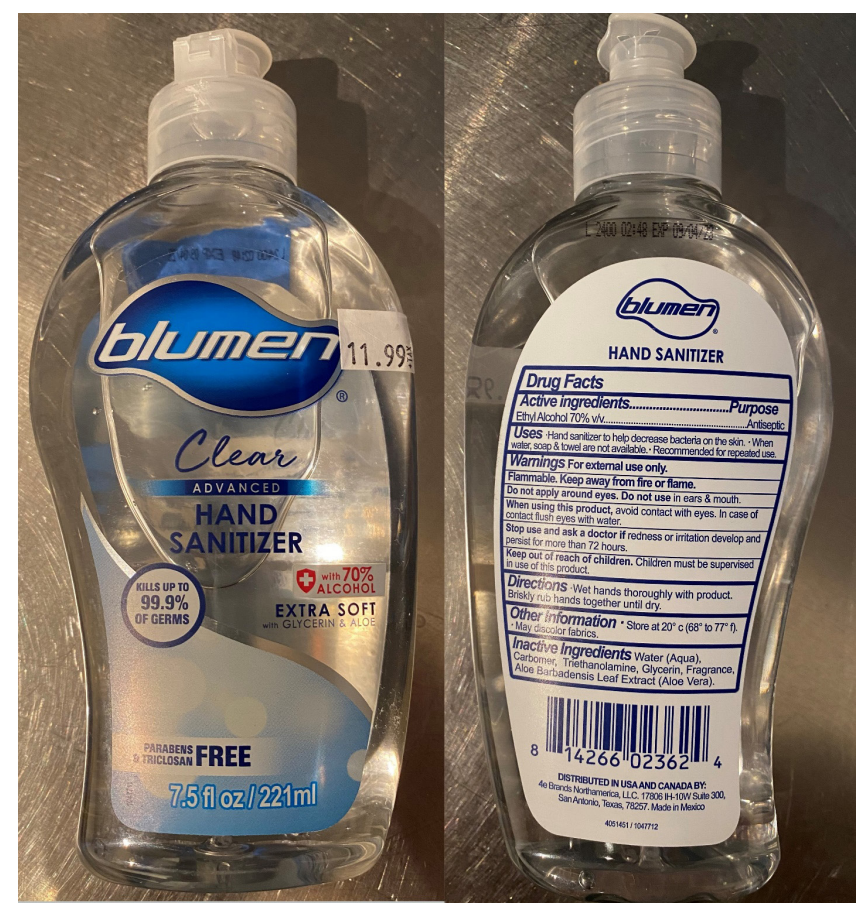

Fig. 3. Image of hand sanitiser confirmed to contain methanol. care providers missing esoteric or unexpected diagnoses and requires even further vigilance on the part of clinicians.

An additional diagnostic dilemma demonstrated by this case is the difficulty and absolute necessity of having a clear, definitive diagnosis of brain death. While in this case, the physical exam and brain perfusion imaging were congruent, not having recognised that this patient's neurologic exam was affected by the mechanism of death could result in misdiagnosis of brain death in a patient with methanol toxicity. As such, health care providers must be aware that brain death cannot be confirmed until the patient is hemodynamically stable and all evidence of metabolic derangement is corrected, and no potential sources of false-negative results exist. In an unreliable examination, such as in this patient, ancillary testing, such as EEG and brain perfusion magnetic resonance imaging (MRI), is crucial to prevent the misdiagnosis of brain death.

\section{CONCLUSION}

In summary, even when exposure history is unclear or seemingly incompatible with methanol poisoning, a broad differential must be considered. Elevated serum osmolarity and metabolic acidosis should raise concerns for non-ethanol alcohol toxicity. Empiric treatment can be started with fomepizole, folate supplementation, and hemodialysis while confirmation laboratory test results are pending. Nevertheless, severe toxicity and delay in the presentation can result in neurologic damage or death. In such cases, patients may be unresponsive, precluding attempts to assess visual acuity. Given that the diagnosis of brain death is contingent on a reliable physical exam, retinal and optic nerve damage should be assumed, and corroborating evidence through EEG and brain perfusion imaging, along with expert neurologic consultation, must be performed before declaring a patient brain dead.

\section{CONFLICT OF INTEREST}

None to declare.

\section{DEFERENCES}

1. Narasimha VL, Shukla L, Mukherjee D, et al. Complicated Alcohol Withdrawal-An Unintended Consequence of COVID-19 Lockdown. Alcohol Alcohol. 2020 Jun 25;55(4):350353. 
70 - The Journal of Critical Care Medicine 2022;8(1)

2. Chiu A, Shepherd K, Shammas B, Itkowitz C. Trump claims controversial comment about injecting disinfectants was 'sarcastic.' Washington Post. Accessed August 3, 2020. https:// www.washingtonpost.com/nation/2020/04/24/disinfectantinjection-coronavirus-trump/

3. Davis LE, Hudson D, Benson BE, Easom LAJ, Coleman JK. Methanol Poisoning Exposures in the United States: 19931998. . J Toxicol Clin Toxicol. 2002;40(4):499-505.

4. Fill MM, Seger DL, Dunn JR, Schaffner W, Jones TF. Notes from the Field: Intoxication and Deaths Associated with Ingestion of a Racing Fuel and Carbonated Soft Drink Mixture- Tennessee, January 2016. MMWR Morb Mortal Wkly Rep. 2016 Jun 10;65(22):585-6.

5. Methyl Alcohol (Methanol) | NIOSH | CDC. Published February 24, 2020. Accessed July 19, 2020. https://www.cdc.gov/niosh/ topics/methyl-alcohol/default.html

6. FDA Says Avoid These 9 Hand Sanitisers That Contain Toxic Methanol. Healthline. Published July 29, 2020. Accessed December 29, 2020. https://www.healthline.com/health-
Available online at: www.jccm.ro news/fda-says-avoid-9-hand-sanitizers-that-contain-toxicmethanol

7. Ashurst JV, Nappe TM. Methanol Toxicity. In: StatPearls. StatPearls Publishing; 2020. Accessed July 19, 2020. http:// www.ncbi.nlm.nih.gov/books/NBK482121/

8. Mégarbane B. Treatment of patients with ethylene glycol or methanol poisoning: focus on fomepizole. Open Access Emerg Med. 2010;2:67-75.

9. Gonda A, Gault H, Churchill D, Hollomby D. Hemodialysis for methanol intoxication. Am J Med. 1978;64(5):749-758.

10. Theobald J, Lim C. Folate as an Adjuvant Therapy in Methanol Poisoning. Nutrition in Clinical Practice. 2019;34(4):521-527.

11. Grzybowski A, Zülsdorff $M$, Wilhelm H, Tonagel F. Toxic optic neuropathies: an updated review. Acta Ophthalmol. 2015;93(5):402-410.

12. Research C for DE and. FDA Updates on Hand Sanitisers with Methanol. FDA. Published online July 17, 2020. Accessed July 19, 2020. https://www.fda.gov/drugs/drug-safety-andavailability/fda-updates-hand-sanitizers-methanol 